\title{
Revisit Eosinopenia as a Biomarker for Diagnosis of Sepsis: A Meta-Analysis
}

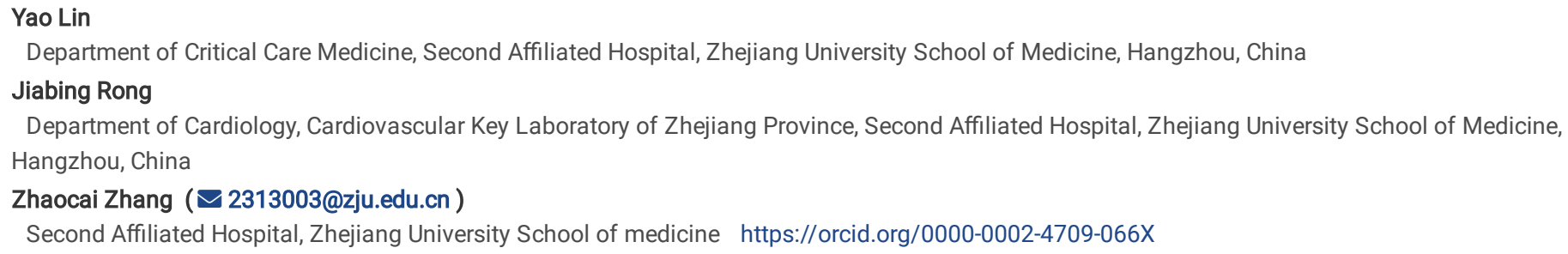




\section{Abstract}

Background

Sepsis is a life-threatening and time-critical medical emergency, therefore, the early diagnosis of sepsis is essential to timely treatment and favorable outcomes for patients susceptible to sepsis. Eosinopenia has been identified as a potential biomarker of sepsis in the past decade. So, we performed a metaanalysis to assess the diagnostic efficacy of eosinopenia for sepsis.

\section{Method}

We searched PubMed, EMBASE, Web of Science, and Cochrane Central Register of Controlled Trials CENTRAL databases to identify studies that met the inclusion criteria. Two authors performed data extraction independently. The pooled outcomes were calculated by TP (true positive), FP (false positive), FN (false negative), TN (true negative) by using bivariate meta-analysis model in STATA 14.0 software.

\section{Results}

Seven studies were included in the present study with a total number of 3842 subjects. The pooled sensitivity, specificity, positive likelihood ratio, negative likelihood ratio and diagnostic odds ratio were 0.66 (95\% Cl [0.53-0.77]), 0.68 (95\% Cl [0.56-0.79]), 2.09 (95\% Cl [1.44-3.02]), 0.49 (95\% Cl [0.34-0.71]) and 4.23 $(95 \% \mathrm{Cl}[2.15-8.31])$, respectively. The area under the summary receiver operator characteristic curve (SROC) was 0.73 (95\% Cl [0.68-0.76]). Meta-regression analysis revealed that no single parameter accounted for the heterogeneity of pooled outcomes. For each subgroup of different eosinopenia cutoff values (50, $40,<=25,100$ ), the sensitivity was $0.61,0.79,0.57,0.54$, and the specificity was $0.61,0.75,0.83,0.51$, respectively.

\section{Conclusions}

Our findings suggest that eosinopenia shows no superiority in the diagnosis of sepsis. Further large clinical trials are needed to re-evaluate eosinopenia as a biomarker of sepsis.

\section{Background}

As "a life-threatening organ dysfunction caused by a dysregulated host response to infection"[1], sepsis has been recognized as a major threat to global health causing millions of deaths per year globally according to WHO, and a true time-critical medical emergency susceptible to death [2]. Therefore, an accurate and quick diagnostic testing is essential to improve outcomes in the patients with sepsis. Although working as the gold standard for identifying infectious conditions, microbial culture has obvious limitations such as time-consuming delay and and considerable false negative results[3]. Thus, seeking timely, sensitive and specific biomarkers has become the research focus in this field, with C reaction protein (CRP), procalcitonin (PCT) and white blood cell count as potential candidates, while the efficiency is far below satisfaction $[3,4]$

Eosinophils, comprising 1\%-3\% of circulating leukocytes, play a role in host defense against helminths and the propagation of allergic conditions, and are considered as an integral part of immune and inflammatory network, and homeostatic regulation as well in recent years [5]. The reduction of circulating eosinophil count (namely eosinopenia) in response to acute infection was firstly described in 1893 by Zappert et al.[6], and is now acknowledged as a signal of acute infection $[7,8]$. The feasibility of eosinopenia in diagnosis of sepsis was firstly tested by Abidi et al, who showed that eosinopenia is good at discriminating between non-infection and infection, but is rather weak in differentiating sepsis from systemic inflammatory response syndrome (SIRS) [9]. Given that there is a significant correlation between sepsis and eosinopenia, several studies carried out in addressing the value of eosinopenia to diagnose sepsis recently [9-15] and to predict the prognosis of sepsis[16-18] with pretty much inconsistence. With emerging of new studies on eosinopenia as a marker of sepsis, we determine to perform the present meta-analysis to reevaluate whether eosinopenia is a good biomarker for diagnosis of sepsis.

\section{Methods}

\section{Study design and Literature search}

The present meta-analysis was performed according to the Preferred Reporting Items for Systematic Reviews and Meta-Analysis (PRISMA) statement[19]. The protocol for this meta-analysis is available in PROSPERO (CRD42020197664). Studies that investigated the diagnostic efficacy of eosinopenia for sepsis were searched for inclusion. Discrepancies were resolved by the consensus of all authors.

A comprehensive electronic search of the PubMed, EMBASE, Web of Science, and Cochrane Central Register of Controlled Trials CENTRAL databases was performed. Then we reviewed the reference lists of included articles to obtain additional relevant articles. No language restriction or publication date restrictions were applied, and the date of our search was until 9 July 2020.

We used the keywords "sepsis" and "eosinopenia" to search articles, and the process of study selection was schematically presented in the PRISMA flow diagram. The detailed search strategy is showed as in Suppl. Table 4.

\section{Selection criteria}

Studies were selected based on the following inclusion criteria: (1) enough data to calculate the outcome data (true positive(TP), false positive(FP), false negative(FN), true negative(TN)); (2) the gold standard for diagnosis of sepsis was defined in the study; (3) prospective or retrospective study design; (4) only 
adult patients were involved. The literature was excluded if they are (1) reviews, case reports, editorials and animal experiments; (2) only healthy people were used as controls.

\section{Data extraction}

Two authors (LY, RJB) independently collected data referring to study and patient characteristics. A third author (ZZC) independently assessed these data in case of inter-reviewer discrepancies. The extracted information from each study included first author name, year of publication, country, study design, sample size, control patients, cutoff value, prevalence, male, mean age, timing of eosinophils counts assessment, reference standard and outcome data (TP, FP, FN, TN). For studies providing multiple eosinopenia cutoff values, the outcome data of all cutoff values were extracted.

\section{Quality assessment}

The methodological quality of the included studies was tested with the QUADAS-2 tool by two authors. QUADAS-2 consists of four sections: patient selection, index text, reference standard, and flow and timing[20]. The included studies were ranked as low risk, high risk, or unclear risk. We performed the quality assessment using Review Manager (RevMan), version 5.3 (Cochrane Collaboration)

\section{Statistical analysis}

The pooled sensitivity, specificity, positive likelihood ratio (PLR), negative likelihood ratio (NLR), diagnostic odds ratio (DOR), AUC, and corresponding 95\% credible interval $(\mathrm{Cl})$ were calculated by TP, FP, FN, TN using a bivariate regression model using STATA 14.0 software. Deek's funnel plot was used to detect publication bias, with $P<0.05$ indicating publication bias. A Fagan plot was assembled for the visual presentation of diagnostic performance.

We used Spearman correlation coefficient to detect threshold effects by using Meta-DiSc software (version 1.4), and $P$ value $<0.05$ indicates a significant threshold effect. We used $\mathrm{I}^{2}$ to describe the heterogeneity in the meta-analysis. $\mathrm{I}^{2}$ value $\geq 50 \%$ is considered as a significant heterogeneity. Then, the possible sources of heterogeneity were explored by conducting a meta-regression analysis and sensitivity analysis. The examined parameters in meta-regression included cutoff ( $\geq 50 /<50$ ), control patient (SIRS/non-infection), sample size $(\geq 200 /<200$ ), type of study (prospective/retrospective), country (Europe and America/others). A sensitivity analysis was conducted to examine the influence of each study on the meta-analysis, by calculating the pooled outcomes after omitting one study at a time. The impact of eosinopenia cutoff used in each study was further investigated by performing a subgroup analysis.

\section{Result}

\section{Included studies}

7 studies that represent 12 trials were included in this meta-analysis (using different cutoff values in the same studies was regarded as different trials), with a total number of 3842 subjects. Among which, 1152 were diagnosed as sepsis, while the rest of 2690 were considered as non-sepsis. A summary of the characteristics (first author name, year of publication, country, study design, sample size, control patients, cutoff value, prevalence, male, mean age, timing of Eosinophils counts assessment) of the 7 included studies is outlined in Table 1 . The data used for the construction of the $2 \times 2$ table was presented in Suppl. Table 1, along with the cutoff values of each study. A flow diagram was assembled to describe the details of the study selection process (Fig. 1) 
Table 1

The characteristics of included studies

\begin{tabular}{|c|c|c|c|c|c|c|c|c|c|c|c|c|}
\hline Author & Year & Country & Study design & $\begin{array}{l}\text { Clinical } \\
\text { Setting }\end{array}$ & $\begin{array}{l}\text { Reference } \\
\text { standard }\end{array}$ & $\begin{array}{l}\text { Severity } \\
\text { of } \\
\text { sepsis }\end{array}$ & $\begin{array}{l}\text { Sample } \\
\text { Size }\end{array}$ & Prevalence & Male & $\begin{array}{l}\text { Mean/ } \\
\text { median } \\
\text { Age }\end{array}$ & $\begin{array}{l}\text { Control } \\
\text { Patients }\end{array}$ & $\begin{array}{l}\mathrm{Cu} \\
(\mathrm{C} \epsilon \\
/ \mathrm{m}\end{array}$ \\
\hline \multirow[t]{2}{*}{ Abidi } & \multirow[t]{2}{*}{2008} & \multirow[t]{2}{*}{ Morocco } & \multirow[t]{2}{*}{ Prospective } & \multirow[t]{2}{*}{ ICU } & \multirow[t]{2}{*}{ ACCP/SCCM } & \multirow{2}{*}{$\begin{array}{l}\text { Sepsis; } \\
\text { severe } \\
\text { sepsis; } \\
\text { septic } \\
\text { shock }\end{array}$} & \multirow[t]{2}{*}{177} & \multirow[t]{2}{*}{$120 / 177$} & \multirow[t]{2}{*}{ 101/177 } & \multirow[t]{2}{*}{42} & $\begin{array}{l}\text { SIRS + } \\
\text { Negative }\end{array}$ & 50 \\
\hline & & & & & & & & & & & SIRS & 40 \\
\hline Anand & 2016 & India & Prospective & ICU & ACCP/SCCM & $\begin{array}{l}\text { severe } \\
\text { sepsis }\end{array}$ & 170 & $125 / 170$ & $107 / 170$ & 52 & SIRS & 50 \\
\hline Garnacho & 2014 & Spain & Prospective & ICU & ACCP/SCCM & $\begin{array}{l}\text { severe } \\
\text { sepsis; } \\
\text { septic } \\
\text { shock }\end{array}$ & 160 & $117 / 160$ & $79 / 160$ & 63 & SIRS & 25 \\
\hline Moura & 2011 & Brazil & Retrospective & ICU & ACCP/SCCM & $\begin{array}{l}\text { Sepsis; } \\
\text { severe } \\
\text { sepsis; } \\
\text { septic } \\
\text { shock }\end{array}$ & 282 & $99 / 282$ & & $58.6+-20$ & $\begin{array}{l}\text { SIRS + } \\
\text { Negative }\end{array}$ & 10( \\
\hline Lavoignet & 2016 & France & Retrospective & ED & ACCP/SCCM & $\begin{array}{l}\text { Sepsis; } \\
\text { severe } \\
\text { sepsis; } \\
\text { septic } \\
\text { shock }\end{array}$ & 692 & $125 / 692$ & 0.45 & $59+-17.2$ & $\begin{array}{l}\text { Non- } \\
\text { infection }\end{array}$ & $\begin{array}{l}\frac{10(}{50} \\
\frac{10}{10}\end{array}$ \\
\hline López & 2010 & Spain & Retrospective & ICU & ACCP/SCCM & $\begin{array}{l}\text { Sepsis; } \\
\text { severe } \\
\text { sepsis; } \\
\text { septic } \\
\text { shock }\end{array}$ & 244 & $55 / 244$ & $165 / 244$ & $54.4+-19.8$ & $\begin{array}{l}\text { Non- } \\
\text { infection }\end{array}$ & 10 \\
\hline Shaaban & 2010 & USA & Prospective & ICU & ACCP/SCCM & $\begin{array}{l}\text { Sepsis; } \\
\text { severe } \\
\text { sepsis; } \\
\text { septic } \\
\text { shock }\end{array}$ & 68 & $31 / 68$ & $33 / 68$ & 68 & $\begin{array}{l}\text { SIRS + } \\
\text { noSIRS }\end{array}$ & 50 \\
\hline
\end{tabular}

Footnotes: ACCP/SCCM: American College of Chest Physicians/Society of Critical Care Medicine; Negative: negative results of bacterial culture; SIRS: syster response syndrome; ICU: intensive care unit

\section{Quality assessment}

We used the QUADAS-2 tool to evaluate the quality of included studies, and results are illustrated in Fig. 2 and summarized in Suppl. Figure 1. High risk of bias was indicated in the section of index test, as 5 studies did not use pre-defined cutoff values to calculate sensitivity and specificity, but rather used the optimal ones, and 1 study did not mention whether it used a pre-defined value. When considering applicability concerns, 3 studies showed potential problems in patient selection.

\section{Publication bias}

The Deeks' funnel plot indicated no evidence of publication bias $(P=0.65)$ (Suppl. Figure 2).

\section{Outcomes}

The pooled sensitivity of eosinopenia for the prediction of sepsis was $0.66(95 \% \mathrm{Cl}[0.53-0.77])$ and the pooled specificity was 0.68 (95\% $\mathrm{Cl}[0.56-0.79])$ (Fig. 3). The pooled PLR was 2.09 (95\% Cl [1.44-3.02]) and the pooled NLR was 0.49 (95\% Cl [0.34-0.71]) (Suppl. Figure 3). The diagnostic odds ratio was $4.23(95 \% \mathrm{Cl}[2.15-8.31])$ (Suppl. Figure 4) and the area under the curve (AUC) was $0.73(95 \% \mathrm{Cl}[0.68-0.76])$ (Fig. 4). Fagan's nomogram for likelihood ratios showed that by using eosinopenia to diagnose sepsis, the post-probability increased to $47 \%$ and decreased to $17 \%$, when the pre-test probability was set at $30 \%$ (Suppl. Figure 5).

The Spearman correlation coefficient $(\rho=0.056, P=0.863)$ indicated that no threshold effect existed.

We used $\mathrm{I}^{2}$ to measure the heterogeneity in our meta-analysis. For the pooled sensitivity, specificity, PLR, NLR, DOR, the $\mathrm{I}^{2}$ values were $94.32 \%, 97.32 \%, 91.75 \%$, $92.12 \%, 100 \%$, which indicates a significant heterogeneity in the majority of analysis. To determine the sources of heterogeneity, we brought cutoff, control patient, sample size, study design, country into meta-regression analysis (Fig. 5 and Suppl. Figure 6), and the results showed that the examined covariates could not account for the heterogeneity. We also conducted a sensitivity analysis to detect the influence of each study on the pooled DOR (Fig. 6) and overall outcomes (Suppl. Table. 2). This Leave-one-out analysis indicated that no single study exerted significant influence on the results of the meta-analysis. 
As the cutoff values varied greatly in these included trials, we performed a cutoff subgroup analysis. The results are shown in Suppl. Table. 3 . For eosinopenia cutoff values $(50,40,<=25,100)$, the sensitivity was $0.61,0.79,0.57,0.54$, and the specificity was $0.61,0.75,0.83,0.51$, respectively.

\section{Discussion}

Sepsis is common and life-threatening in intensive care unit, and early diagnosis and early treatment are essential for the favorable outcome of patients with sepsis. Multiple biomarkers, especially PCT and CRP, have been widely studied for early diagnosis of sepsis in past decade, showing only moderate diagnostic performance [4]. Many other novel biomarkers, such as neutrophil CD64, presepsin, IL-27, cfDNA and miRNAs, also have been intensely examined, showing no definite and satisfactory diagnostic efficacy individually, while combination may provide better sensitivity and specificity[21].

Eosinopenia has been identified as a sign of acute infection for a long time[6-8] and its correlation with sepsis was firstly reported by Abidi et.al in 2008[22]. Eosinopenia is a response to acute inflammation, displaying a decreasing number of eosinophils in circulation, an accumulation of eosinophils at the inflammatory sites, and an inhibition of eosinophils production in bone marrow[23]. Eosinophils are increasingly recognized to play a critical role in modulating local and systemic immune and inflammatory responses[5]. Given that sepsis is accompanied by dysregulated immune responses and inflammatory cascades, it is reasonable to regard eosinopenia as an indication of pathophysiological status during sepsis. In the past decade, eosinopenia has aroused much attention in its diagnostic and prognostic values for bacterial infection and sepsis, with some studies showing promising results.[10-15, $17,18,24,25]$ However, the results of currently available studies on the diagnostic performance of eosinopenia are quite different, which is manifested by a range of sensitivity from $23.2-92.5 \%$ and a range of specificity from $28.57-91 \%$ [9-15]. Therefore, we conducted a meta-analysis to integrate all results from different studies in order to get the pooled outcomes

The present meta-analysis covers studies from 2008 to 2016 on the value of eosinopenia for diagnosis of sepsis in adults, which has not been performed previously. Our primary finding is that eosinopenia is not a good biomarker for the diagnosis of sepsis, as shown the area under the SROC curve was 0.73 , the DOR was 4.23 , and the pooled sensitivity and specificity was 0.66 and 0.68 respectively. Eosinopenia is inferior to the two most widely used biomarkers, PCT and CRP, when being used to diagnose sepsis. According to a latest meta-analysis of the use of PCT and CRP for the diagnosis of sepsis, PCT exhibited an AUROC of 0.85 , a DOR of 12.5 , a sensitivity of 0.80 and a specificity of 0.77 , and CRP exhibited an AUROC of 0.73 , a DOR of 6.89 , a sensitivity of 0.80 and a specificity of $0.61[26]$. Similar result has been found by Jose Garnacho-Montero and his colleagues when compared the diagnostic value of eosinopenia to CRP and PCT in critically ill patients with suspicion of sepsis[11].

However, after a further examination of the literature used for this meta-analysis, we found that there is significant statistical heterogeneity, which has a potential causal relationship with country, sample size, study designs, control setting, and cut-off values etc. Firstly, the 7 included studies were conducted in 7 different countries (suppl. table 2) from Asia, Africa, Europe, and America, where medical levels and ethnic characteristics vary greatly. Secondly the sample size ranges from 68 to 692 as we know that inappropriate sample size may contribute to an unreliable result. However, according to the results of the sensitivity analysis, no individual study that was removed could significantly change the pooled DOR estimated by the remaining studies. Thirdly, we included all observation studies (prospective and retrospective), and it is universally acknowledged that prospective studies have a higher credibility than retrospective studies. Fourthly, some included studies assessed only SIRS patients as controls while the other studies assessed SIRS patients and infected patients without SIRS as controls or just use non-infectious patients as controls (without determining whether they have SIRS). It is more difficult to differentiate sepsis from SIRS in clinical practice because of the similar clinical signs. Fifthly, the cutoff values of eosinopenia among these included trials range from 10 to 100 $\left(\right.$ cells $/ \mathrm{mm}^{3}$ ), which may also be the sources of heterogeneity, and then we performed a subgroup analysis. When using the cutoff value $=40$ cells $/ \mathrm{mm}^{3}$, eosinopenia showed the best diagnostic performance with a sensitivity of 0.79 and a specificity of 0.75 , although the insufficient number of included trials in some subgroups is a big limitation of the subgroup analysis. However, no individual factors could account for the heterogeneity except the parameter of country can interpret the heterogeneity of specificity alone. Most likely there are undiscovered factors contribute to the heterogeneity. Furthermore, according to this meta-regression analysis, study design exerted no observable influence on pooled sensitivity and specificity, while selecting SIRS patients as controls exactly exhibited lower sensitivity and specificity, although there is no statistical significance.

To further explore the potential value of eosinopenia for diagnosis of sepsis in clinical practice, we constructed a Fagan nomogram, which showed that testing eosinopenia could help increase the post-probability to $47 \%$ and reduce the post-probability to $17 \%$, with setting a pre-test probability of $30 \%$. In summary, our results did not support eosinopenia as a clinically useful tool for diagnosis of sepsis.

\section{Limitation of the study}

Firstly, the number of included studies is small, in spite of the extensive search of literature. Secondly, a significant heterogeneity exists in our analysis, and we find no single factor to account for it. Thirdly, these included studies span from 2008 to 2016, and was conducted in 7 different countries across 4 continents, which involves complicated factors such as healthcare system, detection sensitivity, antibiotic utilization, and ethnic characteristics.

\section{Implications for future researches}

Because of the internal heterogeneity and small number of including studies, our results show only weak association between eosinopenia and the pathophysiological status of sepsis. Future researches are supposed to focus on the following two aspects: (1) whether eosinopenia could contribute to the diagnosis of sepsis caused by some specific pathogens; (2) whether eosinopenia show a significant advantage in the era of sepsis-3.0, given that the studies included in our meta-analysis were almost conducted before the emergence of sepsis-3.0 criteria[1].

\section{Conclusion}


Collectively, our results reveal little possibility of eosinopenia as a good biomarker for sepsis due to its low sensitivity and specificity. This condition could be due to the insufficient number of included studies, thus more comprehensive clinical studies should be included to make a definite conclusion. Moreover, future studies can focus on the combination of several biomarkers instead of one single biomarker to diagnose sepsis.

\section{Abbreviations}

TP: True positive

FP: False positive

FN: False negative

TN: True negative

95\%Cl: 95\% credible interval

PLR: Positive likelihood ratio

NLR: Negative likelihood ratio

DOR: Diagnostic odds ratio

AUC: Area under the curve

SROC: Summary receiver operator characteristic

WHO: World Health Organization

CRP: C reaction protein

PCT: Procalcitonin

SIRS: Systemic inflammatory response syndrome

ICU: Intensive care unit

cf-DNA: Cell-free plasma DNA

QUADAS-2: Quality Assessment of Diagnostic Accuracy Studies

\section{Declarations}

\section{Ethics approval and consent to participate}

Not applicable.

\section{Consent for publication}

Not applicable. The manuscript does not contain any personal data in any form.

\section{Availability of data and materials}

All data generated during this study are included in this published article and its supplementary information files.

\section{Competing interests}

The authors declare that they have no competing interests.

\section{Funding}

This research received no specific grant from any funding agency.

\section{Authors' contributions}

LY had full access to all of the data in the study and takes responsibility for the integrity of the data and the accuracy of the data analysis. LY and RJB contributed to the study design, data collection, interpretation, and statistical analysis. LY contributed to the manuscript preparation. ZZC contributed to the study supervision and manuscript revision. All authors read and approved the manuscript. 
Not applicable.

\section{References}

1. Shankar-Hari M, Phillips GS, Levy ML, Seymour CW, Liu VX, Deutschman CS, Angus DC, Rubenfeld GD, Singer M: Developing a New Definition and Assessing New Clinical Criteria for Septic Shock: For the Third International Consensus Definitions for Sepsis and Septic Shock (Sepsis-3). JAMA 2016, 315(8):775-787.

2. Reinhart K, Daniels R, Kissoon N, Machado FR, Schachter RD, Finfer S: Recognizing Sepsis as a Global Health Priority - A WHO Resolution. The New England journal of medicine 2017, 377(5):414-417.

3. Carrigan SD, Scott G, Tabrizian M: Toward resolving the challenges of sepsis diagnosis. Clinical chemistry 2004, 50(8):1301-1314.

4. Perner A, Gordon AC, De Backer D, Dimopoulos G, Russell JA, Lipman J, Jensen J-U, Myburgh J, Singer M, Bellomo R et al: Sepsis: frontiers in diagnosis, resuscitation and antibiotic therapy. Intensive Care Med 2016, 42(12):1958-1969.

5. Chusid MJ: Eosinophils: Friends or Foes? J Allergy Clin Immunol Pract 2018, 6(5):1439-1444.

6. Zappert JJZKM: Ueber das vorkommen der eosinophilen zellen in menschlichen blute. 1893, 23:227-308.

7. Bass DA, Gonwa TA, Szejda P, Cousart MS, DeChatelet LR, McCall CEJTJoci: Eosinopenia of acute infection: production of eosinopenia by chemotactic factors of acute inflammation. 1980, 65(6):1265-1271.

8. Gil H, Magy N, Mauny F, Dupond JL: [Value of eosinopenia in inflammatory disorders: an "old" marker revisited]. Rev Med Interne 2003, 24(7):431-435.

9. Abidi K, Khoudri I, Belayachi J, Madani N, Zekraoui A, Zeggwagh A, Abouqal R: Eosinopenia is a reliable marker of sepsis on admission to medical intensive care units. Critical Care 2008, 12(2).

10. Anand D, Ray S, Bhargava S, Srivastava LM, Garg A, Gafoor I, Singh R, Dhar D: Exploration of eosinopenia as a diagnostic parameter to differentiate sepsis from systemic inflammatory response syndrome: Results from an observational study. Indian Journal of Critical Care Medicine 2016, 20(5):285290.

11. Garnacho-Montero J, Huici-Moreno MJ, Gutierrez-Pizarraya A, Lopez I, Antonio Marquez-Vacaro J, Macher H, Manuel Guerrero J, Puppo-Moreno A: Prognostic and diagnostic value of eosinopenia, C-reactive protein, procalcitonin, and circulating cell-free DNA in critically ill patients admitted with suspicion of sepsis. Critical Care 2014, 18(3).

12. Lavoignet CE, Le Borgne P, Slimani H, Forato M, Kam C, Kauffmann P, Lefebvre F, Bilbault P: [Relevance of eosinopenia as marker of sepsis in the Emergency Department]. Rev Med Interne 2016, 37(11):730-734.

13. López de Toro Martín Consuegra I, Sánchez Casado M, Rodríguez Villar S, Raigal Caño A, López Reina Torrijos P, Velasco Ramos A, Sánchez Rodríguez P, Cabezas Martín H: [Evaluation of eosinopenia as an infection marker in critical care patients]. Med Intensiva 2010, 34(4):246-253.

14. Moura EB, Maia MO, Araújo Neto JA, Amorim FF: Relevance of eosinopenia as an early sepsis marker. Critical Care 2011, $15: 11$.

15. Shaaban H, Daniel S, Sison R, Slim J, Perez G: Eosinopenia: Is it a good marker of sepsis in comparison to procalcitonin and C-reactive protein levels for patients admitted to a critical care unit in an urban hospital? Journal of critical care 2010, 25(4):570-575.

16. Merino CA, Martínez FT, Cardemil F, Rodríguez JR: Absolute eosinophils count as a marker of mortality in patients with severe sepsis and septic shock in an intensive care unit. Journal of critical care 2012, 27(4):394-399.

17. Tinoco-Sánchez M, Suárez-Cuenca JA, Rubio-Guerra AF: Usefulness of eosinopenia as prognostic marker of severity in sepsis. Medicina Interna de Mexico 2017, 33(5):572-579.

18. Varghese J, Devadas K, Cyriac R, Vinayakumar N, Hareendran A, Iqbal A: Low eosinophil count, A Predictor of 28 day mortality in a cohort of cirrhosis patients with sepsis. Journal of Gastroenterology and Hepatology 2019, 34:495.

19. Liberati A, Altman DG, Tetzlaff J, Mulrow C, Gøtzsche PC, loannidis JPA, Clarke M, Devereaux PJ, Kleijnen J, Moher D: The PRISMA statement for reporting systematic reviews and meta-analyses of studies that evaluate health care interventions: explanation and elaboration. J Clin Epidemio/ 2009, 62(10):e134.

20. Whiting PF, Rutjes AWS, Westwood ME, Mallett S, Deeks JJ, Reitsma JB, Leeflang MMG, Sterne JAC, Bossuyt PMM: QUADAS-2: a revised tool for the quality assessment of diagnostic accuracy studies. Annals of internal medicine 2011, 155(8):529-536.

21. Sandquist M, Wong HR: Biomarkers of sepsis and their potential value in diagnosis, prognosis and treatment. Expert Rev Clin Immuno/ 2014, 10(10):13491356.

22. Abidi K, Khoudri I, Belayachi J, Madani N, Zekraoui A, Zeggwagh AA, Abouqal R: Eosinopenia is a reliable marker of sepsis on admission to medical intensive care units. Critical care (London, England) 2008, 12(2):R59.

23. Bass DA: Behavior of eosinophil leukocytes in acute inflammation. II. Eosinophil dynamics during acute inflammation. J Clin Invest 1975, 56(4):870-879.

24. Karakonstantis S, Gryllou N, Papazoglou G, Lydakis C: Eosinophil count (EC) as a diagnostic and prognostic marker for infection in the internal medicine department setting. Romanian Journal of Internal Medicine 2019, 57(2):166-174.

25. Lavoignet CE, Le Borgne P, Chabrier S, Bidoire J, Slimani H, Chevrolet-Lavoignet J, Lefebvre F, Jebri R, Sengler L, Bilbault P: White blood cell count and eosinopenia as valuable tools for the diagnosis of bacterial infections in the ED. European journal of clinical microbiology \& infectious diseases : official publication of the European Society of Clinical Microbiology 2019, 38(8):1523-1532.

26. Tan M, Lu Y, Jiang H, Zhang L: The diagnostic accuracy of procalcitonin and C-reactive protein for sepsis: A systematic review and meta-analysis. $J$ Cell Biochem 2019, 120(4):5852-5859. 
Figures

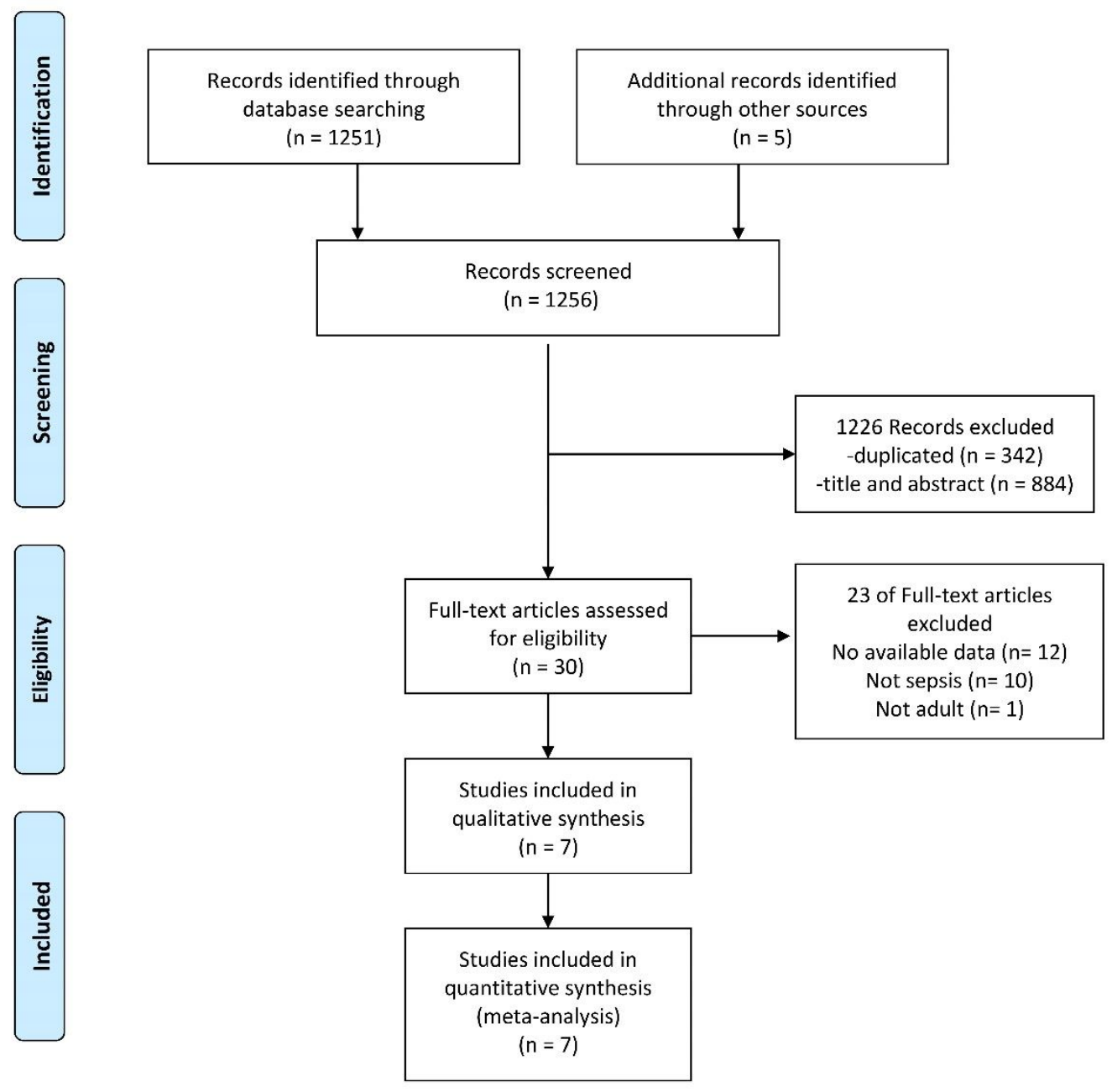

Figure 1

PRISMA Flow diagram

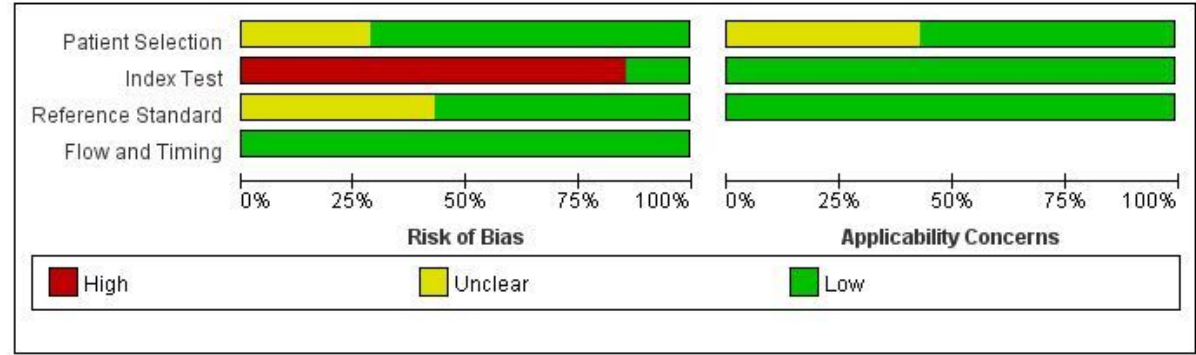

Figure 2

QUADAS2 for quality assessment 

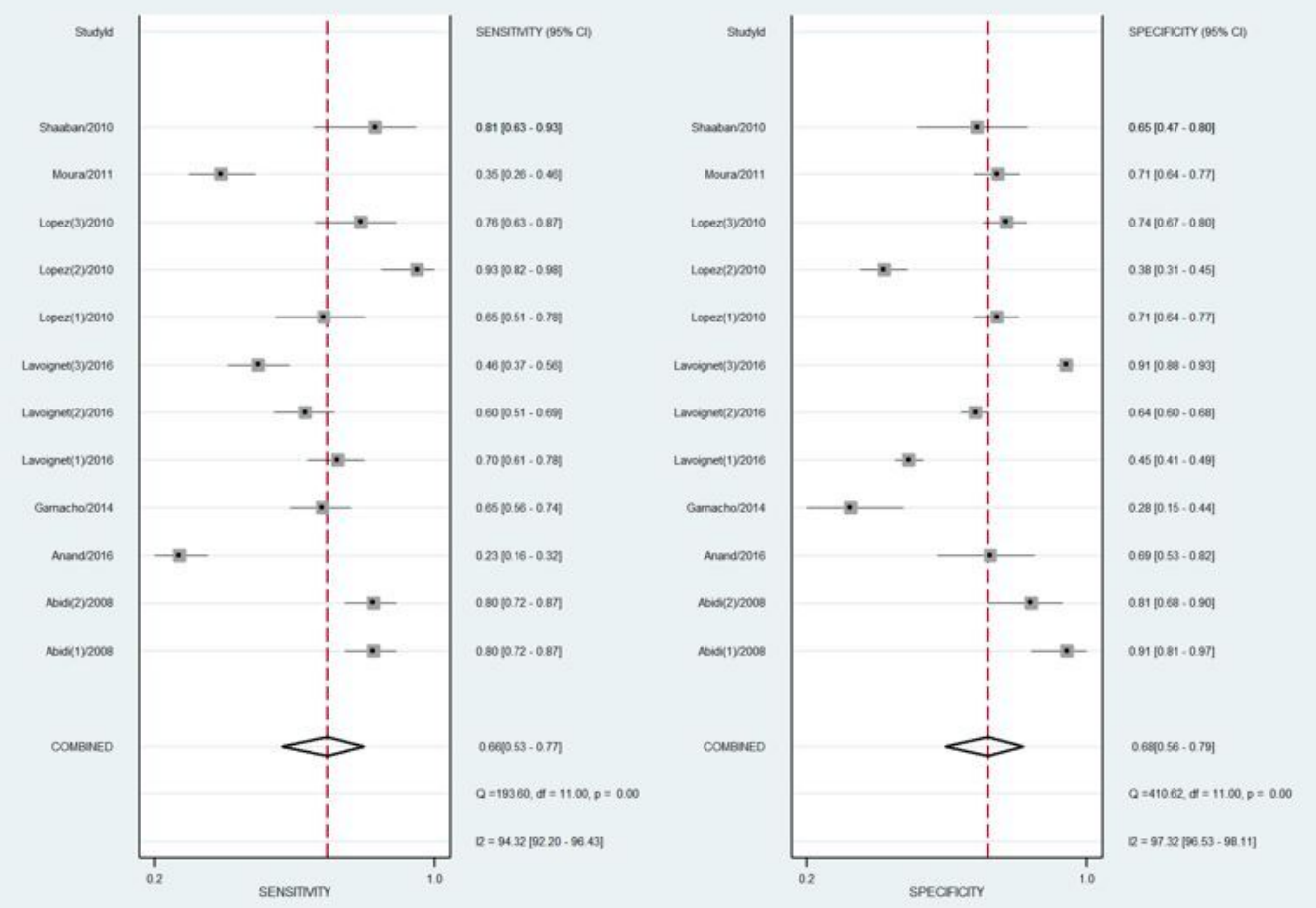

Figure 3

Forest plots of the pooled sensitivity and specificity for eosinopenia in diagnosing sepsis.

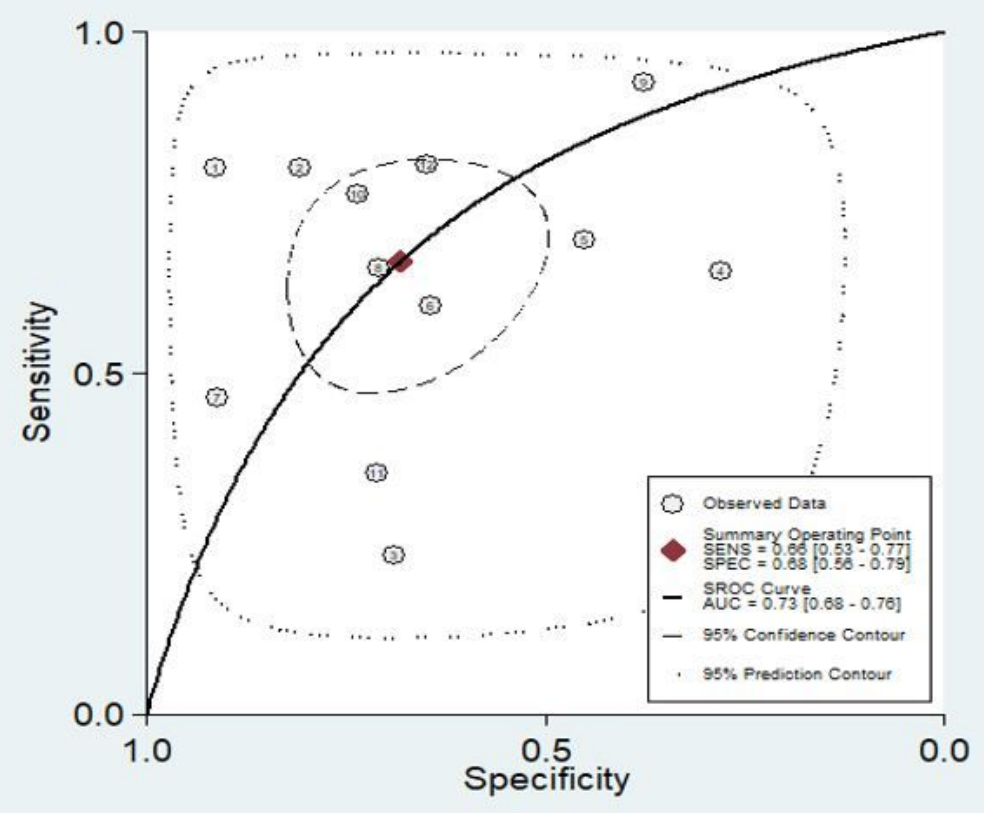

Figure 4

Summary receiver operator characteristic plots with $95 \% \mathrm{Cl}$ of sensitivity against specificity of eosinopenia for diagnosing sepsis. 


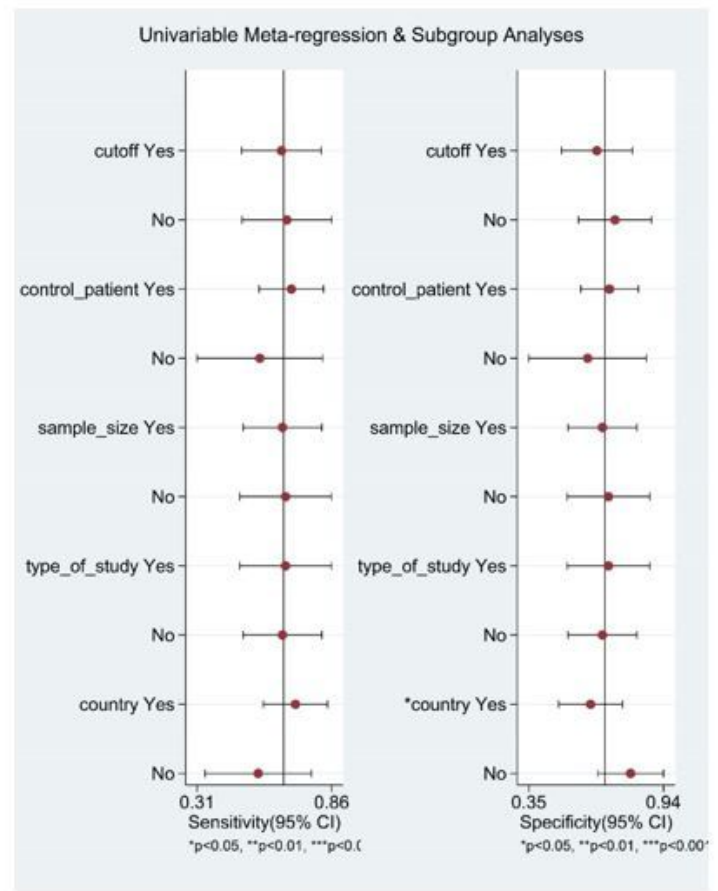

\section{Figure 5}

meta-regression analysis. legend: Yes/No represents that: cutoff ( $\geq 50 /<50)$, control patient (SIRS/non-infection), sample size ( $\geq 200 /<200)$, type of study (prospective/retrospective), country (Europe and America/others).

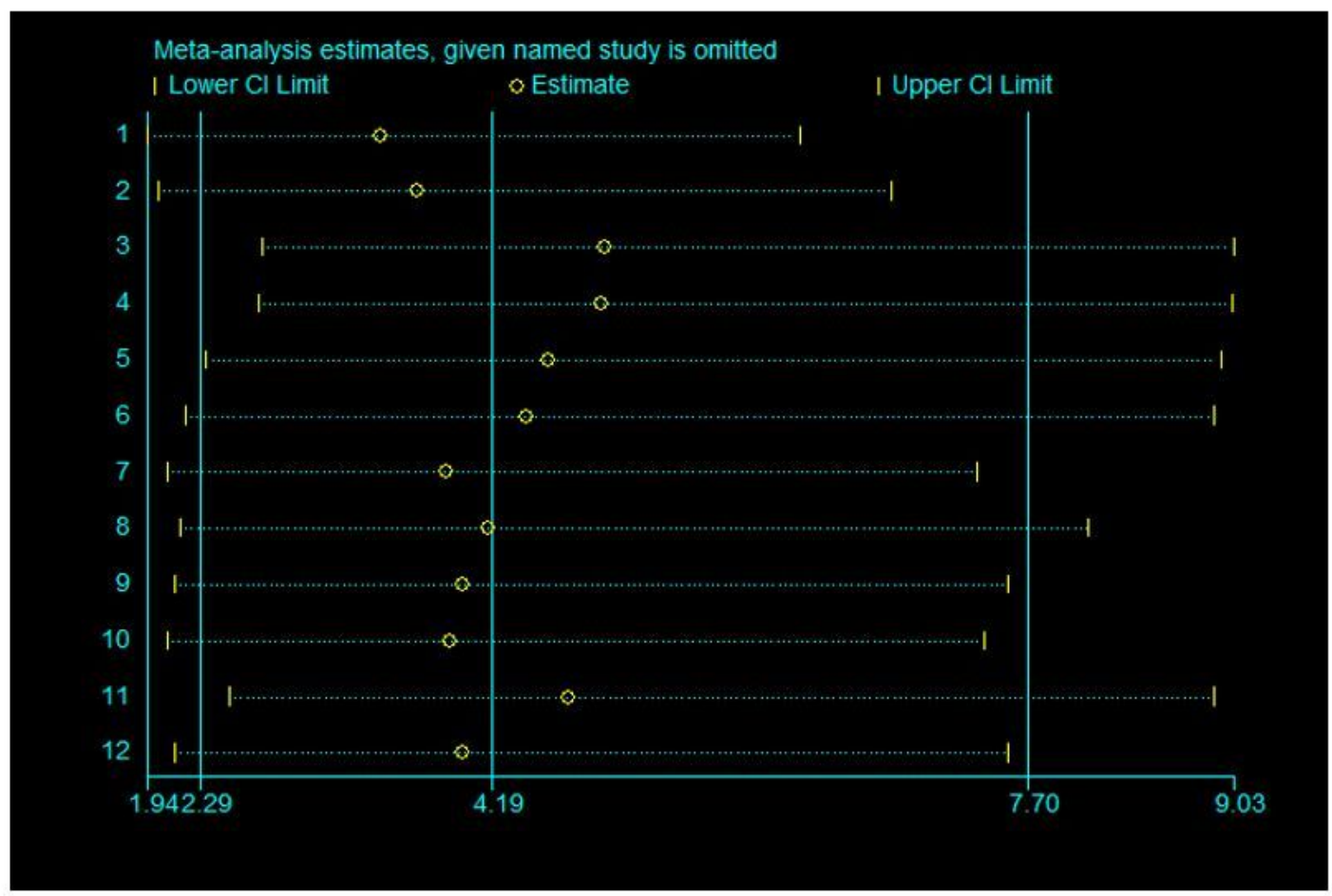

\section{Figure 6}

Sensitivity analysis

\section{Supplementary Files}

This is a list of supplementary files associated with this preprint. Click to download. 
- Suppl.table4searchstrategy.docx

- Suppl.table3cutoffsubgroupanalysis.docx

- Suppl.table2sensitivityanalysis.docx

- Suppl.table1datafor2x2.docx

- Suppl.fig.6metaregressionsummary.tif

- Suppl.fig.5fagan.png

- Suppl.fig.4DORforest.png

- Suppl.fig.3PLRNLRforest.png

- Suppl.fig.2Deekspubbias.png

- Suppl.fig.1qualityassessmentsummary.png 